\title{
A role for microRNAs in the Drosophila circadian clock
}

\author{
Sebastian Kadener ${ }^{1,2,5}$ Jerome S. Menet ${ }^{1,3}$ Ken Sugino, ${ }^{1}$ Michael D. Horwich, ${ }^{3,4}$ Uri Weissbein, ${ }^{2}$ \\ Pipat Nawathean, ${ }^{1,3,6}$ Vasia V. Vagin, ${ }^{3,4,7}$ Phillip D. Zamore, ${ }^{3,4}$ Sacha B. Nelson, ${ }^{1}$ \\ and Michael Rosbash ${ }^{1,3,8}$
}

${ }^{1}$ National Center for Behavioral Genomics and Department of Biology, Brandeis University, Waltham, Massachusetts 02454, USA; $^{2}$ The Department of Biological Chemistry, The Alexander Silberman Institute of Life Sciences, The Hebrew University of Jerusalem, Edmond J. Safra Campus, Givat-Ram, Jerusalem 91904, Israel; ${ }^{3}$ Howard Hughes Medical Institute, University of Massachusetts Medical School, Worcester, Massachusetts 01605, USA; ${ }^{4}$ Department of Biochemistry and Molecular Pharmacology, University of Massachusetts Medical School, Worcester, Massachusetts 01605, USA

Little is known about the contribution of translational control to circadian rhythms. To address this issue and in particular translational control by microRNAs (miRNAs), we knocked down the miRNA biogenesis pathway in Drosophila circadian tissues. In combination with an increase in circadian-mediated transcription, this severely affected Drosophila behavioral rhythms, indicating that miRNAs function in circadian timekeeping. To identify miRNA-mRNA pairs important for this regulation, immunoprecipitation of AGO1 followed by microarray analysis identified mRNAs under miRNA-mediated control. They included three core clock mRNAs-clock (clk), vrille (vri), and clockworkorange (cwo). To identify miRNAs involved in circadian timekeeping, we exploited circadian cell-specific inhibition of the miRNA biogenesis pathway followed by tiling array analysis. This approach identified miRNAs expressed in fly head circadian tissue. Behavioral and molecular experiments show that one of these miRNAs, the developmental regulator bantam, has a role in the core circadian pacemaker. S2 cell biochemical experiments indicate that bantam regulates the translation of $c l k$ through an association with three target sites located within the clk 3' untranslated region (UTR). Moreover, clk transgenes harboring mutated bantam sites in their 3' UTRs rescue rhythms of clk mutant flies much less well than wild-type CLK transgenes.

[Keywords: Clk; bantam; circadian rhythms; miRNAs]

Supplemental material is available at http://www.genesdev.org.

Received May 11, 2009; revised version accepted July 27, 2009.

Most organisms use circadian clocks to keep temporal order and anticipate daily environmental changes (Hall 2003). The circadian machinery of higher eukaryotes is proposed to contain interlocking transcription feedback loops, which are accompanied and complemented by intracellular rhythms in abundance, phosphorylation, and nuclear localization of core clock proteins (Hall 2003). In Drosophila, clock (clk) and cycle (cyc) activate the circadian system by promoting transcription of several key clock genes (Allada et al. 1998; Rutila et al. 1998). Three of these target gene products-PER, TIM, and CWO-repress CLK-CYC-mediated transcription in a

Present addresses: ${ }^{5}$ The Department of Biological Chemistry, The Alexander Silberman Institute of Life Sciences, The Hebrew University of Jerusalem, Edmond J. Safra Campus, Givat-Ram, Jerusalem 91904, Israel. ${ }^{6}$ Center for Integrative Genomics, University of Lausanne, Lausanne 1015, Switzerland. ${ }^{7}$ Cold Spring Harbor Laboratory, Howard Hughes Medical Institute, 1 Bungtown Road, Cold Spring Harbor, NY 11724, USA.

${ }^{8}$ Corresponding author.

E-MAIL rosbash@brandeis.edu; FAX (781) 736-3164.

Article published online ahead of print. Article and publication date are online at http://www.genesdev.org/cgi/doi/10.1101/gad.1819509. daily manner (Hardin et al. 1990; Sehgal et al. 1994, 1995; Yu et al. 2006; Kadener et al. 2007; Lim et al. 2007; Matsumoto et al. 2007). The CLK-CYC heterodimer also activates the expression of VRI and PDP1 (Cyran et al. 2003; Glossop et al. 2003), which contribute to the oscillation of $c l k$ mRNA. These direct target gene activators and repressors are proposed to generate highamplitude mRNA oscillations.

The central role of transcriptional feedback loops has been challenged recently by the idea that other modes of regulation could be more important for keeping circadian time. For example, many core clock components undergo circadian phosphorylation (Edery et al. 1994; Yu et al. 2006), and circadian kinase mutants have strong period phenotypes (Rothenfluh et al. 2000; Lin et al. 2002). Moreover, it was demonstrated recently that under certain conditions the cyanobacterial circadian clock can keep time without active transcription (Tomita et al. 2005).

Features of recent experiments are also relevant to nontranscriptional modes of regulation. We recently analyzed a CYC-VP16 fusion protein that increases transcriptional activation of the CLK-CYC dimer (Kadener et al. 
2008). Overexpression of this fusion protein in pacemaker neurons of the fly brain caused short periods ( $22 \mathrm{~h})$ at the molecular and behavioral levels, indicating that transcription is involved in period determination. Moreover, transcription was dramatically increased as measured by luciferase activity: between threefold to fourfold and sixfold depending on the reporter. However, the levels of real CLK target mRNAs experienced an increase of only 1.5 -fold to twofold, suggesting that other levels of regulation may exist (Kadener et al. 2008). One possibility is that translational regulation by microRNAs (miRNAs) may decrease mRNA levels.

miRNAs are small, noncoding RNAs that serve as posttranscriptional regulators of gene expression ( Du and Zamore 2005). Their mechanism of action involves the formation of imperfect hybrids with their target mRNAs, usually by binding to $3^{\prime}$ untranslated regions (UTRs) (Bartel 2009). This leads to mRNA translational repression and increased mRNA turnover. miRNAs associate with target mRNAs as part of a large silencing complex (RNA-induced silencing complex, or RISC), which in Drosophila includes the protein AGO1 (Du and Zamore 2005).

Although miRNAs are emerging as major regulators of developmental and physiological processes, little is known about their role in the nervous system and behavior. Although five reports describe miRNA levels under circadian control in Drosophila, mouse, and Arabidopsis (Cheng et al. 2007; Xu et al. 2007; Yang et al. 2008; Gatfield et al. 2009; Sire et al. 2009), only one demonstrates a miRNA role in the core circadian clock, and this was in the entrainment pathway (Cheng et al. 2007).

To address a wider role of miRNAs in circadian rhythms, we used two approaches. The first, AGO1 immunoprecipitation followed by microarray analysis, identified a population of mRNAs under miRNA control. We found three core circadian mRNAs among them: $c l k$, vri, and cwo. The second identified miRNAs expressed in fly circadian tissue, with circadian cell-specific inhibition of the miRNA biogenesis pathway followed by tiling arrays. The two approaches identified 10 miRNAs potentially involved in circadian rhythms. One of these, the developmental regulator bantam, was then confirmed to have a role in the central circadian clock. Interestingly, bantam had been predicted to regulate the clk 3' UTR (Grun et al. 2005). Indeed, cell culture experiments confirm that bantam regulates the translation of clk via an association with three bantam target sites located within the clk 3' UTR. In combination with the strong effect that mutation of these sites has on circadian rhythmicity in flies, the results demonstrate that bantam regulates clk translation and that this control is an integral part of the circadian clock.

\section{Results}

miRNA biogenesis is necessary for circadian rhythms

To address the role of miRNAs in Drosophila circadian rhythms, we first assayed locomotor activity rhythms in flies with a defective miRNA processing pathway. To affect this pathway specifically in circadian tissues, a UAS-dicer-1 inverted repeat (IR) transgene was combined with a tim-gal4 driver transgene (Tim-DcrIR strain). Dicer-1 is required for miRNA biogenesis in Drosophila (Lee et al. 2004). The expression of the IR for this mRNA should decrease DICER-1 levels and therefore lead to inhibition of miRNA biogenesis. The tim-gal4 driver is known to express in most if not all fly head circadian cells (Stoleru et al. 2004). However, the Tim-DcrIR strain has normal adult locomotor activity rhythms (Supplemental Fig. 1A).

In an attempt to increase the efficacy of the tim-gal4 UAS-dicer-1 IR combination, we added a third transgene; namely, UAS-CYC-VP16. Recent results indicate that UAS-CYC-VP16 significantly increases the transcriptional power of the CLK-CYC dimer and concomitantly the strength of the tim driver (Kadener et al. 2008). To increase even further the dicer-1 knockdown, we performed the behavioral assay at $29^{\circ} \mathrm{C}$; higher temperatures have been shown to increase GAL4-mediated transcriptional activation (Duffy 2002). With these additions, expression of the UAS-dicer 1 IR transgene now dramatically weakened the amplitude of the locomotor activity rhythms compared with the control (Fig. 1A,B). This indicates that miRNAs indeed contribute to the generation, maintenance, and/or output pathways of these adult rhythm phenotypes.

\section{Development of an in vivo method to identify miRNA-regulated $m R N A s$}

To address which mRNA-miRNA pairs might contribute to rhythmicity, we first established an experimental procedure to identify mRNAs under miRNA-mediated regulation in fly heads. To this end, we assayed mRNAs after immunoprecipitation of AGO1 from wild-type fly head extracts. The strategy takes advantage of an excellent monoclonal antibody against Drosophila AGO1 (Miyoshi et al. 2005) and relies on the fact that this is the principal AGO family member in miRNA-containing RISC complexes (Okamura et al. 2004; Forstemann et al. 2007). RNA was isolated from the immunoprecipitated pellet (IP) as well as the initial lysate (input, INP), and then amplified, labeled, and hybridized to expression oligonucleotide microarrays (Drosophila 2.0, Affymetrix) (Fig. 2A).

The immunoprecipitations were performed from fly heads collected at six different circadian time points (Zeitgeber time 3 [ZT3], ZT7, ZT11, ZT15, ZT19, and ZT23). For each sample and probe set that passed the expression threshold (see the Supplemental Material), an enrichment score was averaged from duplicate experiments. It was calculated as the relative degree of association with AGO1; i.e., a ratio between the signal in the IP relative to the INP. A single value (enrichment index [ENR]; highest value from the six time points) was subsequently assigned to each probe set. We then ranked expressed probe sets according to ENR: a higher ranking corresponds to probe sets with higher ENR (IP/INP) values (the highest-ranking mRNA is ranked \#1) (Fig. 2A). 


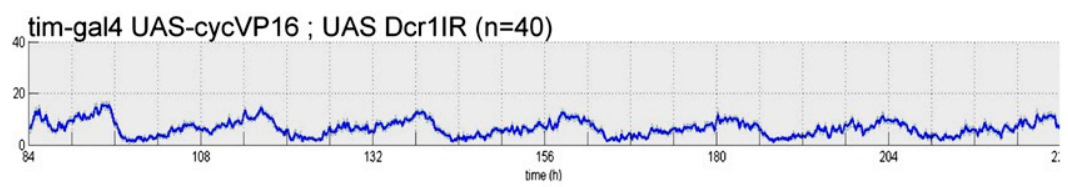

${ }_{40}$ tim-gal4 UAS-cycVP16 $(n=21)$

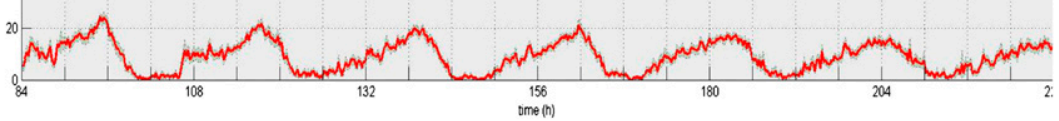

B

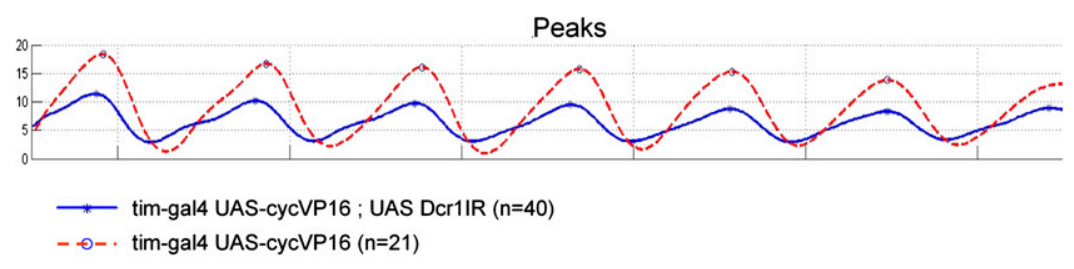

Figure 1. Behavioral characterization of Tim-DcrIR flies. (A) Comparison of circadian locomotor behavior of control flies (tim-gal4; UAS-CYC-VP16; top tracing) and Tim-CYCVP16-Dcr1IR flies (tim-gal4/UAS-CYC-VP16; UAS-Dcr1 IR bottom tracing) in free-running conditions (DD). The experiment was performed at $29^{\circ} \mathrm{C}$. In each case, the behavior is shown in average actograms. $(B)$ Control and Tim-CYCVP16-Dcr1 IR data sets were smoothed with a low-pass filter set with a cutoff of $12 \mathrm{~h}$.
Of the 15 top-ranked mRNAs, 13 are predicted to be miRNA targets, and 10 are predicted to harbor more than one miRNA-binding site by the PicTar algorithm (Fig. 2B; Grun et al. 2005). The probability of obtaining this enrichment by chance is $P=1.9 \mathrm{e}^{-7}$ as calculated from the hypergeometric distribution. Moreover, 77 of the top
100 mRNAs in the ENR ranking contain PicTar predicted miRNA sites in their $3^{\prime}$ UTRs $\left(P=1.3 \mathrm{e}^{-31}\right)$. For a complete ranking, see Supplemental File 1.

To visualize in more detail the relationship between ENR ranking and number of PicTar predicted targets, we calculated and plotted the local density of PicTar
A

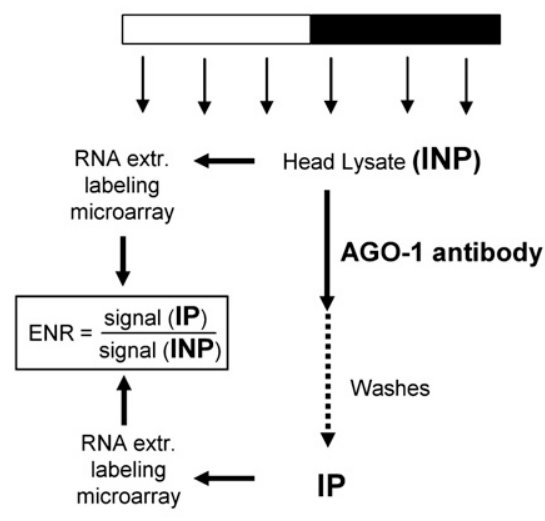

C

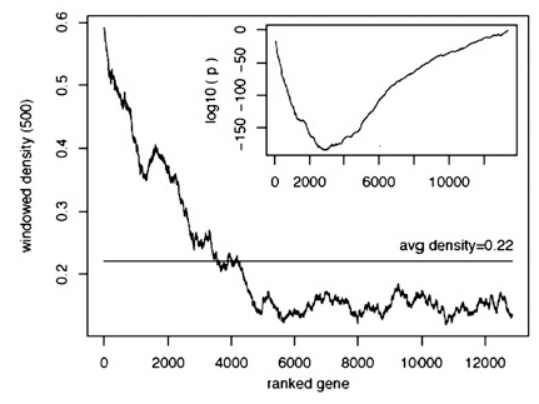

\begin{tabular}{ccc}
\hline RANK & NAME & \# PicTar \\
\hline 1 & BobA & 1 \\
2 & Vang & 8 \\
3 & Mura & 0 \\
4 & Ana & 9 \\
5 & Bip1 & 1 \\
6 & CG14869 & 7 \\
7 & Sinu & 3 \\
8 & Fz4 & 12 \\
9 & Mew & 2 \\
10 & Neur & 10 \\
11 & CG15345 & 0 \\
12 & Brd & 3 \\
13 & BobA & 1 \\
14 & CG15385 & 8 \\
15 & Cyp18a1 & 3 \\
\hline \hline
\end{tabular}

D

\begin{tabular}{cc}
\hline \hline NAME & Ranking \\
\hline \hline$c l k$ & 480 \\
vri & 923 \\
cwo & 1585 \\
\hline tim-RA & 3463 \\
Pdp-1 & 4123 \\
per & 7539 \\
$d b t$ & 8249 \\
$c y c$ & 9217 \\
pdf & 11300 \\
tim-RC & 12553 \\
cry & 12771 \\
\hline \hline
\end{tabular}

Figure 2. Detection of AGO1-associated mRNAs from fly heads. $(A)$ Diagram illustrating the AGO1 IP procedure. For each expressed probe set, an ENR was calculated as a ratio between the expression values in the IP and the INP fractions. The maximum enrichment across samples was used to sort the probe sets in a descending order. This rank order was used in the following calculations. (B) Top 15 enriched mRNAs in the AGO1-IP ranking. "\# PicTar" refers to the number of PicTar predicted miRNA-binding sites in the indicated mRNA. $(C)$ Density of PicTar predicted miRNA targets across the AGO-IP enrichment ranking. The inset represents the enrichment $P$-value for top $\mathrm{X}$ ranking genes, with X moved from 50 to 12,847 with increments of 50. (D) Position in the AGO1-IP enrichment ranking of 10 circadian-relevant mRNAs. 
targets along the ENR ranking with a window of 500 . Higher-ranking probe sets, corresponding to efficiently immunoprecipitated mRNAs, are highly enriched in PicTar targets (Fig. 2C). The plot of the probability of obtaining an enrichment of the top $\mathrm{X}$ probe sets by chance (X ranges from 50 to 12,847 and is plotted against $\mathrm{X}$ in Fig. 2C, inset) indicates that the observed enrichment of PicTar predicted targets in the IP reflects an association of specific mRNAs with AGO1. Additional evidence for the validity of the RISC IP comes from a comparison of ENR ranking with data on miRNA expression levels in fly heads (Ghildiyal et al. 2008): mRNAs with higher enrichment coefficients are predicted to be targeted by more highly expressed miRNAs than mRNAs with higher rankings, which harbor more sites for medium and poorly expressed miRNAs (Supplemental Fig. 2A).

To further validate the immunoprecipitation procedure, we assayed the AGO1-IP from Tim-Dcr IR flies (see above). The experiments were performed at two different time points, ZT3 and ZT15, and the data were processed and ranked as indicated above for the wild-type samples. The density of predicted miRNA targets for the average of these two time points showed a striking decrease in the fraction of miRNA-regulated mRNAs compared with the same two time points from wild-type heads (Supplemental Fig. 2B).

Clk, vri, and cwo are associated with the RISC in fly heads

To determine whether circadian-relevant mRNAs might be subject to miRNA regulation, we examined the AGO1IP data for previously identified cycling genes ( 33 strongly circadian mRNAs identified in two different studies) (Claridge-Chang et al. 2001; McDonald and Rosbash 2001). Despite the presence of two cycling genes (ana and Cyp18a1) among the 15 most enriched probes (Fig. 2B), we did not find a significant enrichment of cycling mRNAs among the top 3000 probes of the AGO1-IP. However, three of the 10 mRNAs encoding canonical central clock components were enriched in the AGO1-IP: clk, vri, and cwo (Fig. 2D); clk and vri mRNAs are particularly enriched.

To determine whether the association of these and other mRNAs with AGO1 is under temporal control, we compared the cycling profiles of the INP and IP mRNA data sets. The vast majority of all probes (including most core circadian components) showed indistinguishable profiles (Supplemental Fig. 3A; data not shown). The temporal profile of clk mRNA, however, is different between the INP and IP samples as a function of time (Supplemental Fig. 3B). In the INP sample, the peak of mRNA abundance is at ZT23, in agreement with previous literature (Cyran et al. 2003; Glossop et al. 2003). However, the peak in the IP sample is a few hours later (Supplemental Fig. 3B). This difference suggests that clk mRNA is most strongly associated with AGO1 during the morning, a few hours after its mRNA abundance peak.
Clk 3' UTR is regulated by the miRNA pathway in $S 2$ cells

To assay further for miRNA translational control of clk mRNA, we tested whether its 3' UTR confers regulation to a luciferase transgene in S2 cells. To this end, we generated reporter constructs containing the actin promoter, a luciferase ORF, and either a control (SV40) 3' UTR, the tim 3' UTR, or the clk 3' UTR. We transfected these reporters into Drosophila S2 cells and measured luciferase levels $48 \mathrm{~h}$ post-transfection. Addition of the clk 3' UTR caused a dramatic decrease in luciferase levels (Fig. 3A). The tim 3' UTR had no effect on luciferase levels, consistent with its failure to associate significantly with AGO1 (Figs. 2D, 3A).

Notably, inhibition of the miRNA pathway by depleting Ago1 via dsRNAi significantly rescued luciferase levels of the clk UTR reporter with no effect on the construct with the tim 3' UTR (Fig. 3B). Control dsRNA targeting either $g f p$ or ago2 (required for siRNA but not miRNA function) (Matranga and Zamore 2007) also had no effect on the clk UTR reporter (Fig. 3B). The data therefore indicate that clk mRNA is repressed by AGO1bound miRNAs in S2 cells as well as fly heads.

\section{Determination of circadian-relevant miRNAs}

To identify miRNA candidates responsible for the regulation of clock core components, we first identified miRNAs expressed in fly head circadian cells. To this end, we searched for an increase in primary miRNAs (primiRNAs) with Drosophila tiling arrays in combination with a tissue-specific knockdown of the miRNA processing pathway. We used the tim-gal4 driver described above in combination with either a UAS-drosha IR or a UASpasha IR transgenic construct (Dietzl et al. 2007). Unlike DICER-1, DROSHA and PASHA function in the nucleus in the initial processing step of miRNA biogenesis and cleave a pri-miRNA primary transcript into an $\sim 70$ nucleotide (nt) pre-miRNA (Du and Zamore 2005). As the tim-gal4 driver restricts inhibition of the pri-miRNA processing machinery to tim-expressing cells, pri-miRNAs expressed in these cells should accumulate (Kadener et al. 2009). We extracted fly head RNA from three different strains collected at two different time points, ZT3 and ZT15: Tim-DroshaIR (tim-gal4; UAS-drosha IR) flies, Tim-PashaIR (tim-gal4; UAS-pasha IR) flies, and a control strain (tim-gal4). For each genotype, the RNA samples from the two different time points were mixed and used to prepare probes for Drosophila tiling arrays. Unlike standard expression arrays, tiling arrays contain highdensity probes for intergenic and intronic regions as well as exonic regions and can therefore be used to identify primiRNAs, which are located in intergenic or intronic regions (Saini et al. 2007).

This approach identified 27 pri-miRNAs expressed in tim-expressing cells (Fig. 4A,B). We confirmed the result for pri-bantam by using a different strategy involving Northern analysis and the depletion of Dicer by RNAi in tim-expressing cells. It showed an accumulation of prebantam in Tim-DcrIR flies. miR-1, in contrast, shows 
A

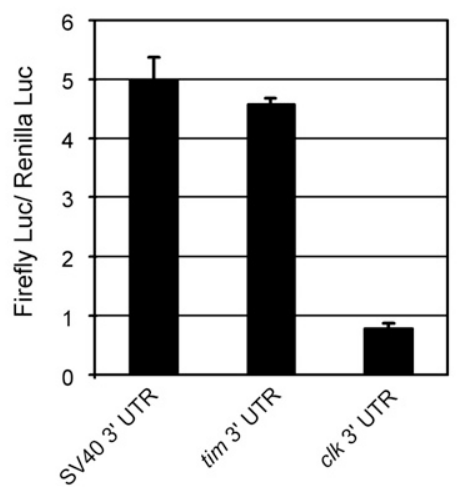

B

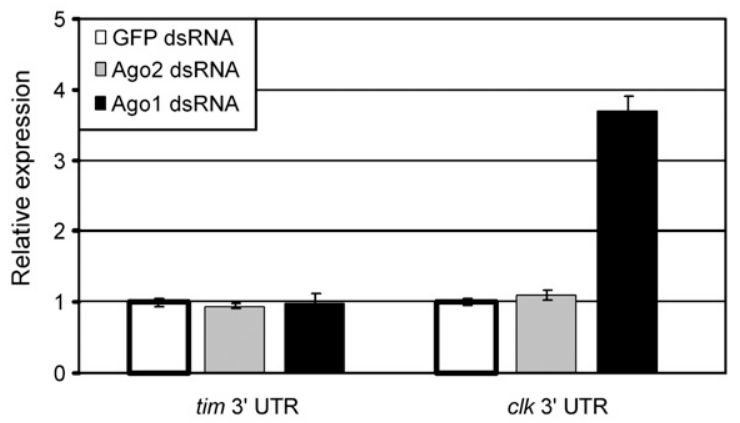

Figure 3. Clk but not tim 3' UTR imparts miRNA-mediated regulation to a luciferase reporter in S2 cells. $(A)$ Effects of different 3' UTRs on the level of expression of a luciferase reporter. Thirty nanograms of pAc-firefly luciferase reporter (containing either the SV40, clk, or tim 3' UTR) were transfected in S2 cells. Forty-eight hours post-transfection, cell extracts were prepared and firefly luciferase activity was measured. In all cases, cotransfection with pCopia-Renilla Luciferase was performed. For each condition, a normalized Firefly/Renilla Luciferase value was obtained and plotted accordingly. A representative experiment is shown. For each condition, two experiments with duplicates were performed. Error bars represent standard error of the mean (SEM). (B) Similar experiment to the one described in $A$ but the S2 cells were treated for $5 \mathrm{~d}$ prior transfection with dsRNA against GFP, Ago1, or ago2. Forty-eight hours posttransfection, cell extracts were prepared, and firefly luciferase activity was measured. The ratios obtained for each dsRNA treatment for the constructs containing the clk and tim 3' UTRs were normalized to the value in the same condition in the SV40 3' UTR. The final value was obtained by setting the value with the addition of the control dsRNA to 1.

little or no precursor accumulation in this strain (Fig. 4C; data not shown). These data confirm that bantam is expressed in circadian cells.
To complement these data, we used a bioinformatics approach to identify candidate miRNAs functioning within fly head circadian tissue. To this end, we scored

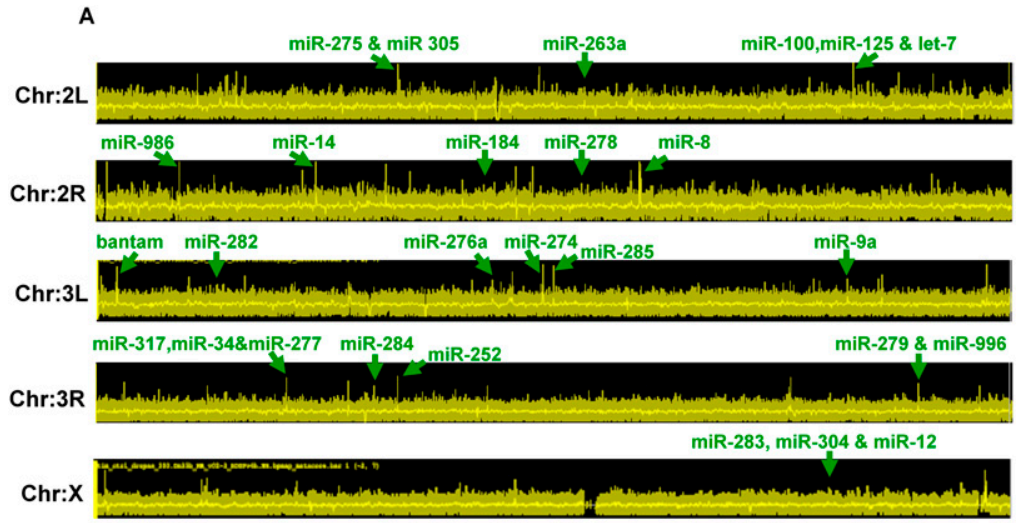

B

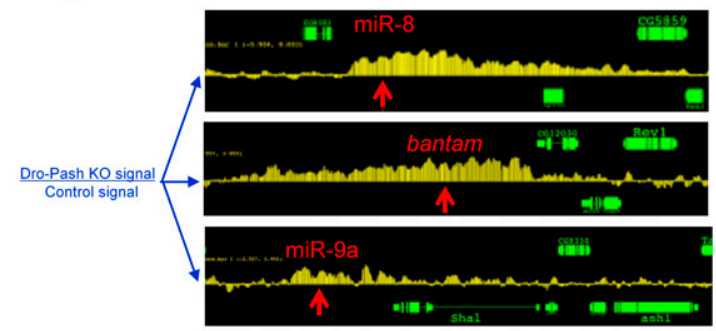

D

\begin{tabular}{ll} 
& Candidates for circadian miRNA \\
\hline \hline Chr:2 & miR-305, miR-8, miR-14 \\
Chr:3 & bantam, miR-9a, miR-34, miR-277, miR-279 \\
Chr:X & miR-12, miR-304 \\
\hline \hline
\end{tabular}

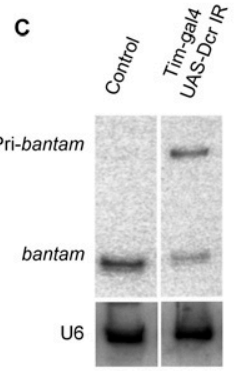

Figure 4. Identification of circadian-relevant miRNAs. (A) Determination of miRNAs expressed in tim cells. The plot represents the difference of expression between control (tim-gal4) and the averaged signal between Tim-DroshaIR and TimPashaIR flies among the $\mathrm{X}$, second, and third chromosomes of Drosophila (see the Materials and Methods for preparation of the samples). Green arrows indicate the coincidence of a significant peak $\left(P\right.$-value $\left.<1 \times 10^{-3}\right)$ with the location of a known miRNA. (B) Close caption of $A$ for three miRNAs: miR-8, bantam, and miR-9a. The signal (in yellow) corresponds to the ratio of signals from Tim-DroshaIR/Tim-PashaIR and control flies. The red arrows indicate the location of the mature miRNA. The genes located around the miRNA sequence are displayed in green and above the yellow signal when their transcription is forward and below when reversed. $(C)$ Detection of Pribantam and mature bantam or U6 snRNA by Northern blot. RNA isolated from control $(y w)$ or Tim-DcrIR (tim-gal4; UAS-dcr-1 IR) fly heads were examined by Northern analysis using a specific probe against bantam or U6 snRNA. $(D)$ List of 10 circadian candidates miRNAs (see the text). 
predicted miRNAs regulating mRNAs that change significantly in their ENR ranking in AGO1 IPs from TimDcrIR flies relative to control flies.

Of the 76 miRNAs with PicTar predicted mRNA targets, the Tim-DcrIR comparison lowered the number to 42 candidates in fly head circadian cells (Supplemental Fig. 4A). Of these, 22 were prime candidates, as these miRNAs were shown previously to be expressed in fly heads at moderate or higher levels by deep sequencing (Supplemental Fig. 4; Supplemental File 2; Ghildiyal et al. 2008).

To lower further the number of candidate circadian miRNAs, we examined the overlap between the two approaches, the predicted miRNAs from the change in the AGO1 IP by the Dicer-1 RNAi construct and the direct assay of pri-miRNAs from drosha(RNAi) or pasha(RNAi) (Fig. 4A). This comparison identified 10 miRNAs (Fig. 4D; Supplemental Fig. 4B).

\section{Overexpression of bantam, but not miR-14, lengthens the core circadian clock}

We next used existing UAS lines to increase expression of two of the 10 candidate miRNAs in tim-expressing tissues (Fig. 4D): miR-14 (Xu et al. 2003) and bantam (Brennecke et al. 2003). Whereas locomotor activity rhythms of flies overexpressing miR-14 were normal (Fig. 5A, right panels), overexpression of bantam with the same driver (Tim-bantam flies) dramatically lengthened circadian period, by almost $3 \mathrm{~h}$ (Fig. 5A, middle panels). To rule out the possibility that this long period was some consequence of the broad expression of the timgal4 driver, we used a $p d f$-gal4 driver to overexpress bantam only in the $18 p d f$-expressing cells (ventral lateral neurons, or LNvs), which likely constitute the key central pacemaker (Renn et al. 1999). Flies with this genetic combination displayed similar long-period rhythms (Fig. 5B).

To further characterize this period lengthening, we assayed the molecular clock more directly. To this end, we added a tim-luciferase reporter gene to the Timbantam strain. Luciferase expression in isolated wings carrying this circadian reporter (McDonald et al. 2001) is robustly rhythmic under LD (light-dark, $12 \mathrm{~h}: 12 \mathrm{~h}$ ) conditions (Kadener et al. 2008). Although wing luciferase rhythms damp significantly after transfer into constant darkness (DD), the first and sometimes even the second DD day can be successfully assayed with $\sim 24$-h periods. Dissected wings from Tim-bantam and control flies were therefore assayed after entrainment, in LD and then for a last day in DD (Fig. 5C).

In agreement with the behavioral effects shown above, overexpression of bantam lengthened circadian period by 2-3 h in DD (Fig. 5C). This result confirms that bantam overexpression affects the molecular machinery within the central pacemaker.

\section{bantam regulates the clk 3' UTR through three evolutionarily conserved binding sites}

Because the Pictar algorithm predicts the presence of a highly conserved site for bantam in the clk 3' UTR (Fig. 6A, red solid line; Supplemental Fig. 5B1; Grun et al. 2005), we tested whether bantam can regulate the $3^{\prime}$ UTR of clk. Indeed, overexpression of bantam in S2 cells decreased the luciferase activity from the clk 3' UTR
Figure 5. Behavioral and molecular effects of bantam overexpression in pacemaker cells. $(A)$ Locomotor behavior of control (tim-gal4), TimmiR14 (tim-gal4; UAS-miR14), and Tim-bantam (tim-gal4; UAS-bantam) flies in DD conditions. The behavior is shown in average actogram (top) and autocorrelation analyses (bottom). The period was calculated for each strain by averaging the periods of individual flies. The error refers to standard error of the mean (SEM). (B) Locomotor behavior of control (UAS-bantam/+) and pdfbantam (pdf-gal4; UAS-bantam) in DD conditions. The behavior is shown in an average actogram. (C) Luciferase recordings from fly wings of control (tim-luc;tim-gal4) and Tim-bantam (tim-luc;timgal4; UAS-bantam) flies using the tim-luciferase reporter. Light timing is indicated by alternating white and gray background areas with white representing the illuminated interval of LD (ZT0-ZT12) and gray representing the dark period (ZT12-ZT24). After $3 \mathrm{~d}$ in LD conditions, the assay was conducted in DD. The results are the average of 24 Tim-bantam and 24 control pairs of fly wings. For each data point, the background (defined as the steady-state value for each strain after $7 \mathrm{~d}$ in DD) was subtracted, and the resulting value was normalized to the maximum for each strain across the experiment.
A
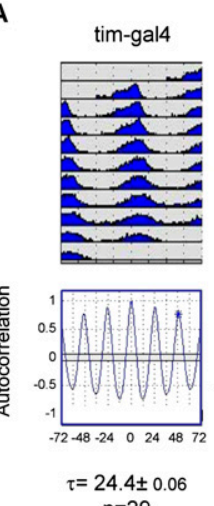

$24.4 \pm 0.06$
$n=29$
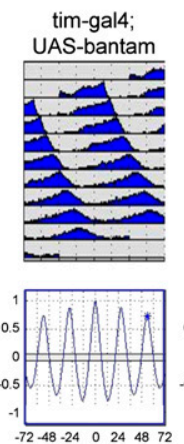

$\tau=26.8 \pm 0.05$ $n=63$

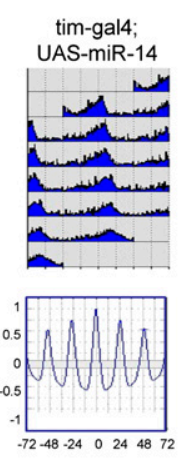

$\tau=\underset{n=38}{24.6 \pm 0.10}$

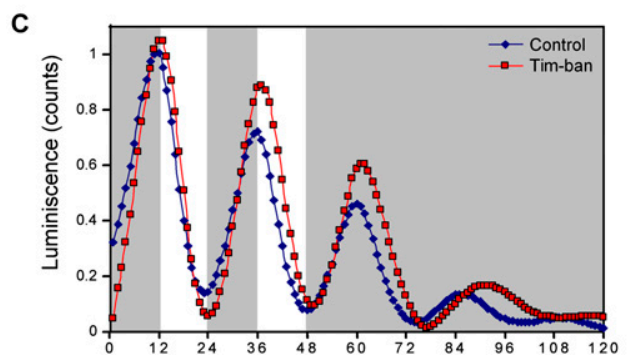

B

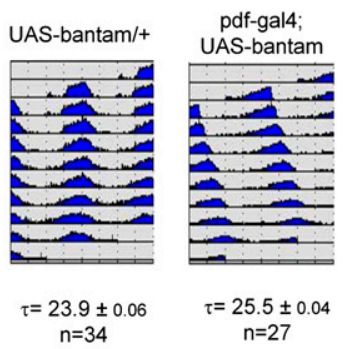


A

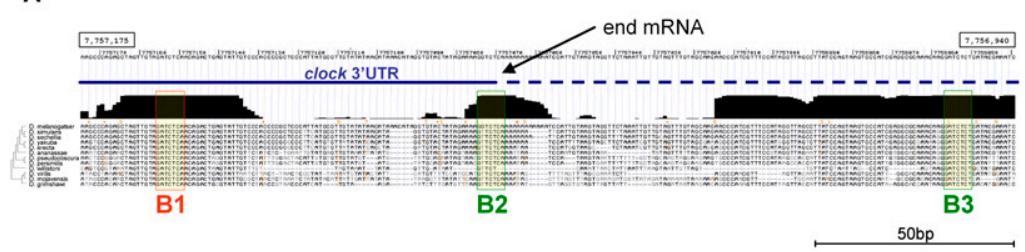

B

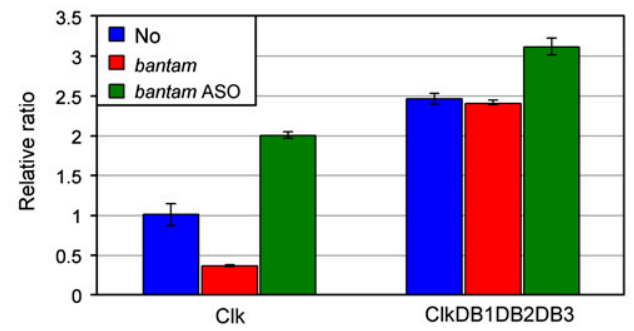

C

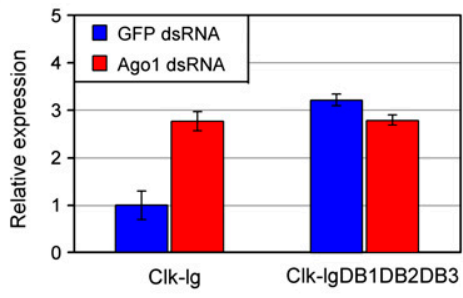

D

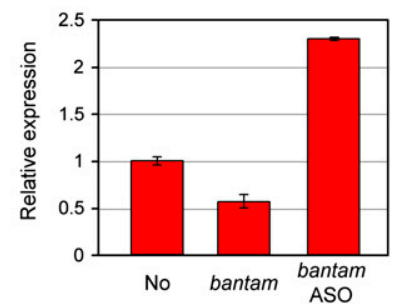

Figure 6. Bantam regulates clk $3^{\prime}$ UTR through three evolutionary binding sites. $(A)$ Representation of clk 3' UTR and conservation between 12 Drosophila species (see the text). Drosophila melanogaster clk 3' UTR sequence (chr3L:7,756,9407,757,175; April 2006 genome assembly) and nucleotide conservation between 12 Drosophila genomes were retrieved using the University of California at Santa Cruz genome Web site. The blue line represents the annotated clk 3' UTR ending at the black arrow (end of clk-sh isoform) (see the text). The red box (B1) indicates the predicted site for the miRNA bantam in the clk 3' UTR. The green boxes (B2 and B3) refer to the two evolutionary bantam sites located downstream from the annotated clk $3^{\prime}$ end (depicted as the dashed blue line). (B) Effect of modulating bantam levels on 3' UTR reporters. S2 cells were transfected with a $3^{\prime}$ UTR luciferase reporter (wild-type clk 3' UTR [left], or mutated bantam sites clk 3' UTR [B1, B2, and B3] [right]) and either an empty plasmid (pAc; first column), a bantam-expressing plasmid (bantam; second column), or 2'-O-methyl anti-bantam cholesterol-conjugated oligos (ban ASO; third column). Renilla luciferase plasmid was cotransfected for normalization. Two days after transfection, luciferase activity was assayed and data were normalized for each reporter to luciferase levels obtained with the empty plasmid. A representative experiment is shown. For each condition,

two experiments with duplicates were performed. Error bars represent standard error of the mean $(\mathrm{SEM})$. (C) Effect of inhibiting the miRNA pathway (by using dsRNA against Ago1) in the levels of clk-lg 3' UTR reporters. The utilized reporters include a wild-type clk$\lg 3^{\prime}$ UTR (left two columns) and a clk-lg 3' UTR in which the bantam sites have been deleted (last two pairs of columns). (D) Effect of modulating bantam levels on the clk-lg 3' UTR reporter. S2 cells were transfected with a clk-lg 3' UTR luciferase reporter and an empty plasmid (pAc; first column), a bantam-expressing plasmid (bantam; second column), or Omethyl anti-bantam cholesterolconjugated oligos (ban ASO; third column). The ratio of Firefly/Renilla luciferase activity was normalized to the luciferase levels obtained with the empty plasmid for each reporter.

reporter by 2.5 -fold (Fig. 6B, left graph, first and second columns), whereas inhibition of bantam (by the introduction of cholesterol-conjugated 2'-O-methyl anti-bantam oligos, ASO) increased twofold the activity of the clk $3^{\prime}$ UTR reporter (Fig. 6B, left graph, first and third columns).

To test whether the effect of bantam on the clk 3' UTR reporter is direct, we deleted the predicted bantambinding site in the clk UTR reporter and assayed for bantam effects on this mutated reporter. Surprisingly, this had no effect, either on basal levels or on the sensitivity of the reporter to changes in expression of bantam (ClkDB1) (Supplemental Fig. 6A,B, respectively). This suggests that the bantam effect on the clk 3' UTR reporter is indirect or through other bantam-binding sites.

Careful inspection of the region downstream from the predicted cleavage and polyadenylation site of the clk $3^{\prime}$ UTR revealed the presence of two additional evolutionarily conserved bantam-binding sites (Supplemental Fig. $5 \mathrm{~B} 2, \mathrm{~B} 3$ ). As the reporter extends 300 base pairs (bp) beyond the predicted end of the $3^{\prime}$ UTR, both additional sites were included (Fig. 6A, areas B2 and B3). Deletion of all three sites from the clk 3' UTR reporter significantly increased S2 cell expression (ClkDB1DB2DB3) (Fig. 6B;
Supplemental Fig. 6A). Moreover, this mutant reporter is insensitive to changes in bantam levels (Fig. 6B; Supplemental Fig. 6B). These results not only demonstrate that bantam directly regulates the clk $3^{\prime}$ UTR, but also suggest that clk extends beyond the annotated cleavage and polyadenylation site. Indeed, dsRNAs targeted against sequences upstream of and downstream from this site indicate that $>70 \%$ of $c l k$ transcripts extend beyond this site (Supplemental Fig. 7A, left column). This is unlikely to be an artifact of the reporter (i.e., too high levels of expression), as dsRNAi targeted against regions downstream from the annotated tim $3^{\prime}$ end has no effect on luciferase activity from a tim $3^{\prime}$ UTR-containing construct (Supplemental Fig. 7A).

3' RACE from fly head RNA indeed identifies two clk $3^{\prime}$ ends (clk-sh and clk-lg) (data not shown). One coincides with the previous annotation (clk-sh) (data not shown), whereas the second extends $\sim 700 \mathrm{bp}$ downstream (clk-lg) (Supplemental Fig. 7B). We also performed RPAs (RNase protection assays) with RNA extracted from fly heads collected at two different time points, ZT2 and ZT14. Two different probes, both complementary to the annotated 3 ' end of clk mRNA, revealed only one strong band with ZT2 RNA and one weak band with ZT14 RNA /typical of 
the clk temporal pattern of expression, ZT2 > ZT14) (data not shown). For both RNAs, the protected fragment extends well beyond the previously annotated clk-sh $3^{\prime}$ end, indicating that $c l k-1 g$ is the predominant if not the only isoform expressed at those time points in fly heads (data not shown). As a track of 12 consecutive adenosines is located $1 \mathrm{nt}$ downstream from the predicted 3' end of the clk-sh isoform (Supplemental Fig. 8B), this isoform may reflect hybridization of the oligo $(\mathrm{dT})$ primer to this A track. Moreover, there is no consensus cleavage and polyadenylation site (AAUAAA) upstream of this predicted clk-sh 3' end (data not shown), in contrast to the strongly conserved AAUAAA sequence $\sim 30$ bases upstream of the 3' end of clk-lg (Supplemental Fig. 8B). We therefore suggest that the clk $3^{\prime}$ end extends $\sim 700$ bases downstream from the annotated site and includes the three characterized bantam sites.

To corroborate that bantam regulates the complete $c 1 k$ 3' UTR, we created a new luciferase reporter that includes the complete $3^{\prime}$ UTR of $c l k-l g$. Indeed, introduction of this $3^{\prime}$ UTR into a luciferase reporter represses activity in an AGO1-dependent manner (Fig. 6D; data not shown). Moreover, reporter activity is strongly affected by bantam levels: Overexpression of this miRNA leads to a twofold decrease, and inhibition of bantam /with a specific ASO) increases reporter activity 2.5-fold (Fig. $6 \mathrm{E})$. Importantly, and in agreement with the results presented above, deletion of the three highly conserved bantam sites in the clk-lg 3' UTR increased basal activity and rendered this reporter insensitive to bantam level manipulations (Fig. 6D; data not shown). This demonstrates that the clk 3' UTR is regulated by bantam through these three evolutionarily conserved binding sites.

\section{The evolutionarily conserved bantam sites are necessary for circadian rhythmicity}

To test whether bantam regulation of clk is important for in vivo circadian rhythmicity, we compared the efficacy of clk transgenes without 3' UTR bantam sites with wildtype clk transgenes to rescue the rhythm defect of a clk mutant strain $\left(c 1 k^{A R}\right)$ (Allada et al. 2003). To this end, we generated flies carrying three different versions of $c l k$ transgenes. The first was a wild-type clk-V5 gene as described previously (Fig. 7A; Kadener et al. 2008). The second was different because it was missing the three described bantam sites (Fig. 7A, three solid lines). The third was also missing a fourth potential bantam site (Fig. 7A, dotted line).

As described previously (Allada et al. 2003), $c 1 k^{A R}$ flies are arrhythmic (Fig. 7B). Introduction of a wild-type copy of the $14.8-\mathrm{kb} c l k-V 5$ transgene significantly rescued the arrhythmic behavior of $c 1 k^{A R}$ flies $149 \%$ and $63 \%$ rhythmic flies for two different insertions of this transgene) (Fig. 7B). However, the $14.8-\mathrm{kb}$ mutant clk-V5 transgenes, without the three or four 3' UTR bantam sites, were much less effective in rescuing locomotor activity rhythms (between $7 \%$ and $26 \%$ rhythmic flies) (Fig. 7B). This was true for a line without the three described bantam sites (Fig. 7B, $\Delta 1-3$, line 2), as well as for three independent lines that also lack the fourth potential bantam site (Fig. 7B, $\Delta 1-4$, lines 4,6,7). Moreover, the few rhythmic flies with the mutant transgenes have significantly weaker rhythms than flies carrying wild-type clk$V 5$ transgenes, as measured by the rhythm index (Levine et al. 2002). The similar results with the two different mutant transgenes suggest that the fourth bantam site is not functional or not important for clk regulation in circadian neurons. Importantly, the poor rescue with the mutant transgenes is not due to a lack of expression; for example, as a consequence of unfavorable insertion sites: clk mRNA levels were indistinguishable between flies carrying wild-type and mutated transgenes, at least under LD conditions and for the two strains assayed: wild-type line 1 and $\Delta 1-4$ line 7 (data not shown). The results demonstrate that bantam regulates clk expression, which is necessary for strong and sustained rhythms.

\section{Discussion}

In the present study, we demonstrate a role for miRNAs in the Drosophila central circadian clock. By performing
Figure 7. The evolutionarily conserved bantam sites are necessary for circadian rhythmicity. (A) Scheme of the clk gene construct used to rescue arrhythmic $c l k^{\mathrm{AR}}$ flies (see the text). The three solid arrows indicate the three evolutionarily conserved bantam sites in the clk 3' UTR. The dotted arrow indicates the fourth potential bantam site in the clk 3' UTR. (B) Table indicating the percentage of rhythmic flies of the different strains. Flies were entrained for $3 \mathrm{~d}$ in LD conditions and then released into DD. Rhythmicity was determined as described in the Materials and Methods. The average RI of rhythmic flies refers to the averaged rhythm index (see the text for details). The differences between flies rescued with control or mutant $c l k$ transgenes were assessed by $t$-test. The obtained $P$-values are the following: for comparison 1 , $P<0.015$; for comparison $2, P<0.0001$; for comparison $3, P<0.015$; for comparison $4, P<0.05$; for comparison $5, P<0.01$; for comparison $6, P<0.05$.
A

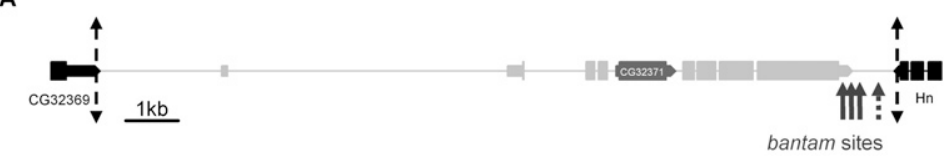

B

\begin{tabular}{|c|c|c|c|}
\hline Genotype & $\%$ Rhythmic & $\begin{array}{l}\text { Average RI of } \\
\text { Rhythmic flies }\end{array}$ & $\begin{array}{c}\text { Period of } \\
\text { Rhythmic flies }\end{array}$ \\
\hline$c l k^{A R} / c l k^{A R}$ & $0 \%(n=27)$ & & \\
\hline$c l k W T($ line 1$) /+; c l k^{A R} / c / k^{A R}$ & $63 \%(n=76)$ & $0.32 \pm 0.048(n=48)^{1,2,3}$ & $23.6 \pm 0.13$ \\
\hline$c l k W T($ line 5$) /+; c l k^{A R} / c l k^{A R}$ & $49 \%(n=45)$ & $0.307 \pm 0.027(n=22)^{4,5,6}$ & $23.5 \pm 0.14$ \\
\hline 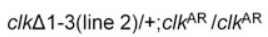 & $21 \%(n=28)$ & $0.248 \pm 0.024(n=10)^{1,4}$ & $23.8 \pm 0.47$ \\
\hline$c l k \Delta 1-4($ line 4$) /+; c l k^{A R} / c / k^{A R}$ & $7 \%(n=14)$ & $0.15(\mathrm{n}=1)$ & 23.5 \\
\hline$c l k \Delta 1-4($ line 6$) /+; c l k^{A R} / c l k^{A R}$ & $17 \%(n=60)$ & $0.212 \pm 0.014(n=10)^{2,5}$ & $24.45 \pm 0.25$ \\
\hline clk $\Delta 1-4$ (line 7$) /+; c l k^{A R} / c l k^{A R}$ & $26 \%(n=34)$ & $0.227 \pm 0.037(n=9)^{3,6}$ & $23.7 \pm 0.25$ \\
\hline
\end{tabular}


AGO1 immunoprecipitation followed by microarray analysis, we identified a population of mRNAs under miRNA control in fly heads. Among them was the master circadian gene clk. In addition, circadian cell-specific inhibition of the miRNA biogenesis pathway followed by tiling arrays identified several miRNAs prominently expressed in circadian tissues. In combination with bioinformatics analyses, the two approaches identified 10 candidate miRNAs involved in circadian rhythms. For one miRNA, the developmental regulator bantam, we present evidence for a direct role in circadian timekeeping. Overexpression of bantam using a circadian cellspecific GAL4 line delays by almost $3 \mathrm{~h}$ the circadian clock at the molecular and behavioral levels. Moreover, we demonstrated that this miRNA regulates $c l k$. This regulation is achieved through three conserved bantam sites in the $3^{\prime}$ UTR of this gene. Two are located downstream from the previously annotated clk mRNA 3' end, and other data indicate that the real clk 3' UTR includes these sites. Genetic experiments in flies demonstrate that the integrity of these three bantam sites is critical for robust circadian rhythmicity. We therefore identified a miRNA-mRNA pair involved in central circadian timekeeping.

This is one of the few studies to use miRNP IP to identify miRNA-regulated mRNAs (Easow et al. 2007; Karginov et al. 2007; Hendrickson et al. 2008; Chi et al. 2009), and may be the first from adult fly tissues. The data fit well with those derived from the PicTar algorithm and should allow a comparison of different miRNA target prediction algorithms.

The second approach for studying specific miRNA expression relies on cell type-specific inhibition of miRNA synthesis pathways in vivo followed by RNA analysis on tiling arrays (Fig. 4). Although very sensitive in identifying many circadian miRNAs, the strategy probably still fails to identify low abundance miRNAs or miRNAs present in small numbers of circadian cells. However, they should be detectable with the same approach, but after a cell purification or cell sorting step. This sensitivity issue is the reason we chose to use the broad tim-gal4 driver rather than the more restricted $p d f$ gal4 driver. Tim-gal4 is expressed strongly in all circadian tissues of the fly head, including circadian neurons, eyes, fat body, and antennae (Hall 2003; Stoleru et al. 2004). This broad expression also explains the strong effect of TIM-Dcr IR on the AGO1 IP enrichment (Supplemental Fig. 2B). Consistent with data indicating that core clock components work similarly in both central (brain) and peripheral tissues (Hall 2003), bantam overexpression slows the clock pace in both locations: in the central brain as demonstrated by behavior (Fig. 5A,B), and in the periphery as demonstrated by luciferase assays (Fig. 5C).

Intersecting the Agol IP data with the tiling array data from Tim-DroshaIR/PashaIR flies as well as with the published fly head miRNA data led to a selection of 10 candidate circadian miRNAs (Fig. 4D; Supplemental File 2). As our analysis only used miRNAs with PicTar target predictions and therefore screened only half of the known miRNA population (Grun et al. 2005), 10 is likely to be an underestimate. On the other hand, of the 27 miRNAs identified as expressed in circadian cells by the TimDroshaIR/PashaIR approach (Fig. 4A), 23 have mRNAs with PicTar predictions in the Ago IP data. This suggests that 10 is not a gross underestimate.

Some of these $10 \mathrm{miRNAs}$ are likely responsible for the decrease in locomotor activity rhythm strength due to inhibition of the miRNA pathway (Fig. 1). It is notable that there are no prior reports of a miRNA contribution to circadian behavior in Drosophila and only a single report in mammals (Cheng et al. 2007). This may be related to the fact that an effect was only manifest at $29^{\circ} \mathrm{C}$ and with the addition of the UAS-CYC-VP16 transgene. The failure to observe a phenotype in Tim-DcrIR flies at $25^{\circ} \mathrm{C}$ may reflect a relatively weak effect of the dicer-1 IR transgene on miRNA expression, consistent with the fact that miRNA biosynthesis is not rate-limiting for miRNAmediated translational regulation (Liu et al. 2007). Nonetheless, it is likely that the lack of a circadian defect in Tim-DcrIR flies is not a consequence of inadequate inhibitory transgene expression. This is because the same strain (Tim-DcrIR) still displays normal rhythms even after increasing the temperature to $29^{\circ} \mathrm{C}$ (data not shown). Moreover, Tim-Dcr seems to strongly downregulate the miRNA pathway, as illustrated by the accumulation of pre-bantam and the substantial change in the AGO1 IP profile (Fig. 4C; Supplemental Fig. 2B).

We therefore suspect that the additional requirement for UAS-CYC-VP16 reflects more than just an increase in UAS- $d$ cr 1 IR expression. It is possible that the transcription and translation of key circadian core components are tightly connected and may buffer each other. Such a regulatory feature could explain why a major increase in transcription, like that caused by the CYC-VP16 transgene, results in only a modest increase in mRNA abundance and probably an even more modest increase in translated protein (Kadener et al. 2008). A comparable explanation posits that inhibition of the miRNA pathway by the UAS-dcr 1 IR transgene leads to an increase in the translation of circadian repressors, which could then decrease circadian transcription. The use of UAS-CYCVP16 as well as $29^{\circ} \mathrm{C}$ might be required to push the system sufficiently far from equilibrium so that pacemaker regulatory mechanisms can no longer compensate for the change in miRNA levels. This type of regulation fits recent data demonstrating that a Drosophila miRNA can function as a buffering agent against environmental perturbations during development (Li et al. 2009). In any case, the observed behavioral defects observed in TimDcrIR-CYCVP16 flies are likely a consequence of downregulation of several circadian-relevant miRNAs (not just bantam).

Our behavioral, genetic, and biochemical evidence indicates that bantam contributes to clk mRNA translational regulation as well as more generally to circadian pacemaker regulation: bantam is highly expressed in circadian tissues, and overexpression with either timgal4 or $p d f$-gal4 significantly lengthens circadian period (Fig. 5A,B). The milder effect of the $p d f$ driver may be due to its lower strength in pacemaker cells relative to 
tim-gal4 (Stoleru et al. 2005) and/or to an additional contribution from non-PDF cells to period determination.

Although the period phenotype could be misleadingdue, for example, to an effect of bantam overexpression on a circadian output pathway-strains with a completely normal central pacemaker do not manifest altered periods, by definition (Hardin et al. 1992; Williams et al. 2001; Inoue et al. 2002). Another possibility-that bantam overexpression renders the circadian neurons sick or unhealthy-would be expected to result in weak rhythms or arrhythmicity rather than in strong rhythms with long periods. The central pacemaker is therefore the most parsimonious explanation, especially because of the good correlation between the behavioral (Fig. 5A) and the molecular data; i.e., the tim-luciferase results (Fig. 5C). Unfortunately, the bantam deletion is embryonic lethal (Brennecke et al. 2003), precluding a straightforward behavioral assay of the null phenotype.

The effect of bantam on clk mRNA translation was aided by the finding that the clk $3^{\prime}$ UTR extends $>700$ bases downstream from its predicted $3^{\prime}$ end. We attribute this error to priming by oligo $(\mathrm{dT})$ within an A-rich region present near this annotated 3' end (Supplemental Fig. 8A). Consistent with this interpretation, a strongly conserved cleavage and polyadenylation site is present near the end of the clk-lg isoform; no obvious site is in the vicinity of the annotated clk 3' end (Supplemental Fig. 8B; data not shown). In addition, our RNA protection data indicate that all fly head clk transcripts extend well beyond the annotated clk 3' end (data not shown). Taken together with the 3' RACE data (Supplemental Fig. 7B), these results demonstrate that the clk 3' UTR is significantly longer than previously indicated. Importantly, two of the three clk 3' UTR bantam-binding sites are located downstream from the annotated $3^{\prime}$ end.

These clk 3' UTR bantam sites appear to be major circadian targets of bantam in flies. First, clk mRNA is strongly associated with RISC (Fig. 2D; Supplemental Fig. 3B). Second, bantam is strongly expressed in the circadian cells, as demonstrated by the accumulation of precursors of this miRNA when Dicer-1, drosha, or pasha was downregulated in fly circadian tissues (Fig. 4A-C). Third, the effect of bantam (lengthening of the circadian period) resembles the period effect observed in flies carrying fewer genomic copies of clk (Allada et al. 1998, 2003), and it is opposite to the period effect observed in flies with additional clk copies (Kadener et al. 2008). Fourth, the three evolutionarily conserved bantam sites are necessary for circadian rhythmicity (Fig. 7B). Nonetheless, the period effect due to bantam overexpression may be due to effects on other mRNAs in addition to clk.

We conclude that miRNAs have a role in the central pacemaker and, more specifically, that bantam regulates the central clock component clk. Whereas previous studies have identified miRNAs relevant to circadian rhythms (Cheng et al. 2007; Xu et al. 2007; Yang et al. 2008; Sire et al. 2009), this one identifies a mRNAmiRNA pair involved in the core timekeeping process. Given the in vivo methods used to study miRNA function (including principally in neuronal tissue), we suspect that they will have a broad impact on the study of miRNAs and their roles in regulating diverse aspects of Drosophila behavior.

\section{Materials and methods}

The sequence of the oligonucleotides used in this study as well as additional methods can be found in Supplemental File 3.

\section{Fly strains}

tim-GAL4, pdf-GAL4, Tim-LUC, UAS-bantam(EP[3]3622), UASmir14, and UAS-CYC-VP16 were described previously (Renn et al. 1999; Kaneko and Hall 2000; Allada et al. 2003; Brennecke et al. 2003; Xu et al. 2003; Kadener et al. 2008). The RNAi lines used were obtained from the Vienna Drosophila RNAi Center (Dietzl et al. 2007).

\section{Identification of AGO-1-associated mRNAs}

AGO-1 immunoprecipitation. Two-hundred-fifty microliters of frozen fly heads were homogenized in $700 \mu \mathrm{L}$ of lysis buffer $(30 \mathrm{mM}$ HEPES KOH at $\mathrm{pH} 7.4,100 \mathrm{mM}$ KAcetate, $2 \mathrm{mM} \mathrm{MgAcetate,}$ $5 \%$ glycerol, $0.1 \%$ Triton X-100, 1 mM EGTA, 5 mM DTT, 0.4 $\mathrm{U} / \mu \mathrm{L}$ RNase OUT [Invitrogen], $1 \times$ EDTA proteinase inhibitor [Roche]). The lysates were incubated for $10 \mathrm{~min}$ on ice and centrifuged at $14,000 \mathrm{rpm}$ for $15 \mathrm{~min}$ at $4^{\circ} \mathrm{C}$. One-hundred microliters of the supernatant were used as input. To the rest of the supernatant $(\sim 500 \mu \mathrm{L}), 1 / 10 \mathrm{vol}$ of anti-AGO-1 antibody (Miyoshi 2005) was added and incubated at $4{ }^{\circ} \mathrm{C}$ with rotation. After $2 \mathrm{~h}, 100 \mu \mathrm{L}$ of protein G plus bead slurry (Calbiochem; previously washed with lysis buffer) were added and incubated for $1 \mathrm{~h}$ at $4^{\circ} \mathrm{C}$ with rotation. The beads were then recovered by gentle centrifugation. The beads were washed five times (beads plus $500 \mu \mathrm{L}$ of lysis buffer were rotated for $5 \mathrm{~min}$ at $4^{\circ} \mathrm{C}$ ), before proceeding to RNA extraction.

Expression microarray probe preparation. Total RNA was extracted using Trizol reagent (Invitrogen). cDNA synthesis was carried out as described in the Expression Analysis Technical Manual (Affymetrix). The cRNA reactions were carried out using the IVT kit (Affymetrix). Affymetrix high-density arrays for Drosophila melanogaster version 2.0 were probed, hybridized, stained, and washed according to the manufacturer's protocol.

Expression array preprocessing. The $\mathrm{R}$ Bioconductor affy package (http://www.bioconductor.org) was used to normalize and calculate summary values from Affymetrix CEL files. CEL files were background-corrected by the "rma2" method, INP and IP groups were quantile-normalized separately, and summary values were calculated together using the "medianpolish" method. Probe sets were filtered by requiring a maximum value of 32 in at least one sample. This yielded 12,847 out of a total of 18,952 probe sets. Affymetrix annotation used in this study (Drosophila_2.na25. annot.csv) was downloaded from the Affymetrix Web site. These data have been deposited at NCBI GEO as SuperSeries (GSE17629).

Analysis for Ago1-IP, TimDcr-Ago1-IP was done using Python (http://www.python.org) and R (http://www.r-project.org) and the in-house library. Analysis scripts are available upon request.

AgoIP rank analysis. Enrichment of IP over INP was calculated as $\log$ fold difference between corresponding samples. The maximum enrichment across samples was used to sort the probe resulting in a descending rank order. This ranking was used in the succeeding calculations. To calculate the enrichment of 
miRNA target genes in IP, PicTar prediction data (Grun et al. 2005) were downloaded from the University of California at Santa Cruz genome browser Web site (http://genome.ucsc.edu), and the density of PicTar predicted miRNA targets was calculated with a window of 500 .

\section{Identification of cell-specific miRNAs}

Tiling arrays. Fly head total RNA was extracted from control (tim-GAL4), Tim-PashaIR (tim-GAL4; UAS-pasha IR), and TimDroshaIR (tim-GAL4; UAS-drosha IR) flies collected at two time points (ZT3 and ZT15) using Trizol reagent (Invitrogen). For each genotype, the RNA samples from these two different time points were then combined and used to prepare probes for Drosophila tiling arrays. cDNA synthesis and labeling were carried out using the wild-type double-stranded cDNA synthesis kit (Affymetrix) and the wild-type dsDNA terminal labeling kit (Affymetrix). Affymetrix Drosophila Tiling 2.0R arrays were probed, hybridized, stained, and washed according to the manufacturer's protocol. One replica of each genotype (two mixed time points) was used.

Tiling array analysis. MAT (model-based analysis of tiling arrays) (Johnson et al. 2006) was used for processing Affymetrix Tiling Array data. The hybridized probes corresponding to the samples Tim-PashaIR and Tim-DroshaIR were compared with the control sample. The genomic regions with significant differences from the comparison $\left(P\right.$-value threshold $\left.10^{-3}\right)$ were manually examined further to determine whether they were located in regions containing any of the 152 previously identified Drosophila miRNAs (Sanger database, http://microrna.sanger.ac.uk). These data have been deposited at NCBI GEO as SuperSeries (GSE17629).

\section{Locomotor behavior}

Male flies were monitored using Trikinetics Drosophila Activity Monitors. Analyses were performed with a signal processing toolbox (Levine et al. 2002). We used autocorrelation and spectral analysis to estimate behavioral cycle durations (periods) and the rhythm index to assess rhythm strength (Levine et al. 2002). For determination of rhythmicity, data from the first $10 \mathrm{~d}$ in DD were used in conjunction with autocorrelation and spectral analysis (Levine et al. 2002).

Plasmids. Copia-Renilla luciferase and pAc-Luc (3' UTR SV40) have been described previously (Kadener et al. 2007). pAc-LuctimRA and pAc-Luc-clk were constructed by amplifying the genomic region containing either the tim-RA or the clk 3' UTR plus 300 bases downstream from the annotated end of the mRNA and ligating these PCR products into pAc-Luc. pAcbantam was constructed by amplifying the sequence from genomic DNA followed by ligation of the PCR product into a pAc His A plasmid (Invitrogen).

Mutations of the bantam sequences in the pAc-luc-clk were introduced by performing overlapping PCR.

\section{S2 cell transfection}

S2 cells were maintained in 10\% FBS (Invitrogen) insect tissue culture medium (HyClone). Cells were seeded in a six-well plate. Transfection was performed at $70 \%-90 \%$ confluence according to company recommendations $(12 \mu \mathrm{L}$ of cellfectin [Invitrogen], $2 \mu \mathrm{g}$ of total DNA). In all experiments, $50 \mathrm{ng}$ of pCopia-Renilla Luciferase plus $30 \mathrm{ng}$ of the Luciferase firefly reporter were used. bantam ASO (3'Cholesterol, 2'OMe bantam antisense oligo) was transfected using Dharmafect 4 (Dharmacon) $2 \mathrm{~d}$ prior to the transfection with the luciferase reporter.

\section{Acknowledgments}

We thank K. Abruzzi, E. Nagoshi, W.F. Luo, and S. Vodala for helpful discussions and suggestions. We also thank K. Palm for administrative assistance. S.K. was a recipient of an HFSP longterm fellowship and currently of an HFSP Career Development Award. J.M. was a recipient of an EMBO long-term fellowship. M.D.H. is funded by an NRSA MD/PhD predoctoral Fellowship from the National Institute of Aging (F30A6030283). The work was partially supported by NIH grants P01-NS44232, GM23549, and P30-NS45713 to M.R.; NIH grants GM62862 and GM65236 to P.D.Z.; and the Lewja Found for Biochemistry and Marie Curie International Reintegration Grant (EU) to S.K.

\section{References}

Allada R, White N, So W, Hall J, Rosbash M. 1998. A mutant Drosophila homolog of mammalian Clock disrupts circadian rhythms and transcription of period and timeless. Cell 93: 791-804.

Allada R, Kadener S, Nandakumar N, Rosbash M. 2003. A recessive mutant of Drosophila Clock reveals a role in circadian rhythm amplitude. EMBO I 22: 3367-3375.

Bartel DP. 2009. MicroRNAs: Target recognition and regulatory functions. Cell 136: 215-233.

Brennecke J, Hipfner DR, Stark A, Russell RB, Cohen SM. 2003. bantam encodes a developmentally regulated microRNA that controls cell proliferation and regulates the proapoptotic gene hid in Drosophila. Cell 113: 25-36.

Cheng HY, Papp JW, Varlamova O, Dziema H, Russell B, Curfman JP, Nakazawa T, Shimizu K, Okamura H, Impey $\mathrm{S}$, et al. 2007. microRNA modulation of circadian-clock period and entrainment. Neuron 54: 813-829.

Chi SW, Zang JB, Mele A, Darnell RB. 2009. Argonaute HITSCLIP decodes microRNA-mRNA interaction maps. Nature 460: 479-486.

Claridge-Chang A, Wijnen H, Naef F, Boothroyd C, Rajewsky N, Young MW. 2001. Circadian regulation of gene expression systems in the Drosophila head. Neuron 32: 657-671.

Cyran SA, Buchsbaum AM, Reddy KL, Lin MC, Glossop NR, Hardin PE, Young MW, Storti RV, Blau J. 2003. vrille, Pdp1, and dClock form a second feedback loop in the Drosophila circadian clock. Cell 112: 329-341.

Dietzl G, Chen D, Schnorrer F, Su KC, Barinova Y, Fellner M, Gasser B, Kinsey K, Oppel S, Scheiblauer S, et al. 2007. A genome-wide transgenic RNAi library for conditional gene inactivation in Drosophila. Nature 448: 151-156.

$\mathrm{Du}$ T, Zamore PD. 2005. microPrimer: The biogenesis and function of microRNA. Development 132: 4645-4652.

Duffy JB. 2002. GAL4 system in Drosophila: A fly geneticist's Swiss army knife. Genesis 34: 1-15.

Easow G, Teleman AA, Cohen SM. 2007. Isolation of microRNA targets by miRNP immunopurification. RNA 13: 1198-1204.

Edery I, Zwiebel LJ, Dembinska ME, Rosbash M. 1994. Temporal phosphorylation of the Drosophila period protein. Proc Nat1 Acad Sci 91: 2260-2264.

Forstemann K, Horwich MD, Wee L, Tomari Y, Zamore PD. 2007. Drosophila microRNAs are sorted into functionally distinct argonaute complexes after production by dicer-1. Cell 130: 287-297.

Gatfield D, Le Martelot G, Vejnar CE, Gerlach D, Schaad O, Fleury-Olela F, Ruskeepaa AL, Oresic M, Esau CC, Zdobnov EM, et al. 2009. Integration of microRNA miR-122 in hepatic circadian gene expression. Genes \& Dev 23: 1313-1326.

Ghildiyal M, Seitz H, Horwich MD, Li C, Du T, Lee S, Xu J, Kittler EL, Zapp ML, Weng Z, et al. 2008. Endogenous 
siRNAs derived from transposons and mRNAs in Drosophila somatic cells. Science 320: 1077-1081.

Glossop NR, Houl JH, Zheng H, Ng FS, Dudek SM, Hardin PE. 2003. VRILLE feeds back to control circadian transcription of Clock in the Drosophila circadian oscillator. Neuron 37: 249-261.

Grun D, Wang YL, Langenberger D, Gunsalus KC, Rajewsky N. 2005. microRNA target predictions across seven Drosophila species and comparison to mammalian targets. PLOS Comput Biol 1: e13. doi: 10.1371/journal.pcbi.0010013.

Hall J. 2003. Genetics and molecular biology of rhythms in Drosophila and other insects. Elsevier Science, New York.

Hardin PE, Hall JC, Rosbash M. 1990. Feedback of the Drosophila period gene product on circadian cycling of its messenger RNA levels. Nature 343: 536-540.

Hardin P, Hall JC, Rosbash M. 1992. Behavioral and molecular analyses suggest that circadian output is disrupted by disconnected mutants in D. melanogaster. EMBO J 11: 1-6.

Hendrickson DG, Hogan DJ, Herschlag D, Ferrell JE, Brown PO. 2008. Systematic identification of mRNAs recruited to argonaute 2 by specific microRNAs and corresponding changes in transcript abundance. PLoS One 3: e2126. doi: 10.1371/ journal.pone.0002126.

Inoue S, Shimoda $M$, Nishinokubi I, Siomi $M$, Okamura $M$, Nakamura A, Kobayashi S, Ishida N, Siomi H. 2002. A role for the Drosophila fragile x-related gene in circadian output. Curr Biol 12: 1331-1335.

Johnson WE, Li W, Meyer CA, Gottardo R, Carroll JS, Brown M, Liu XS. 2006. Model-based analysis of tiling-arrays for ChIPchip. Proc Natl Acad Sci 103: 12457-12462.

Kadener S, Stoleru D, McDonald M, Nawathean P, Rosbash M. 2007. Clockwork Orange is a transcriptional repressor and a new Drosophila circadian pacemaker component. Genes \& Dev 21: 1675-1686.

Kadener S, Menet IS, Schoer R, Rosbash M. 2008. Circadian transcription contributes to core period determination in Drosophila. PLoS Biol 6: e119. doi: 10.1371/journal.pbio. 0060119.

Kadener S, Rodriguez J, Abruzzi KC, Khodor YL, Sugino K, Marr MT II, Nelson S, Rosbash M. 2009. Genome-wide identification of targets of the drosha-pasha/DGCR8 complex. RNA 15: $537-545$

Kaneko M, Hall JC. 2000. Neuroanatomy of cells expressing clock genes in Drosophila: Transgenic manipulation of the period and timeless genes to mark the perikarya of circadian pacemaker neurons and their projections. J Comp Neurol 422: 66-94.

Karginov FV, Conaco C, Xuan Z, Schmidt BH, Parker JS, Mandel G, Hannon GJ. 2007. A biochemical approach to identifying microRNA targets. Proc Natl Acad Sci 104: 19291-19296.

Lee YS, Nakahara K, Pham JW, Kim K, He Z, Sontheimer EJ Carthew RW. 2004. Distinct roles for Drosophila Dicer-1 and Dicer-2 in the siRNA/miRNA silencing pathways. Cell 117: 69-81.

Levine JD, Funes P, Dowse HB, Hall JC. 2002. Signal analysis of behavioral and molecular cycles. BMC Neurosci 3: 1. doi: 10.1186/1471-2202-3-1.

Li X, Cassidy JJ, Reinke CA, Fischboeck S, Carthew RW. 2009. A microRNA imparts robustness against environmental fluctuation during development. Cell 137: 273-282.

Lim C, Chung BY, Pitman JL, McGill JJ, Pradhan S, Lee J, Keegan KP, Choe J, Allada R. 2007. Clockwork orange encodes a transcriptional repressor important for circadian-clock amplitude in Drosophila. Curr Biol 17: 1082-1089.

Lin JM, Kilman VL, Keegan K, Paddock B, Emery-Le M, Rosbash M, Allada R. 2002. A role for casein kinase $2 \alpha$ in the Drosophila circadian clock. Nature 420: $816-820$.
Liu X, Park JK, Jiang F, Liu Y, McKearin D, Liu Q. 2007. Dicer-1, but not Loquacious, is critical for assembly of miRNA-induced silencing complexes. RNA 13: 23242329.

Matranga C, Zamore PD. 2007. Small silencing RNAs. Curr Biol 17: R789-R793. doi: 10.1016/j.cub.2007.07.014.

Matsumoto A, Ukai-Tadenuma M, Yamada RG, Houl J, Uno KD, Kasukawa T, Dauwalder B, Itoh TQ, Takahashi K, Ueda $\mathrm{R}$, et al. 2007. A functional genomics strategy reveals clockwork orange as a transcriptional regulator in the Drosophila circadian clock. Genes \& Dev 21: 1687-1700.

McDonald MJ, Rosbash M. 2001. Microarray analysis and organization of circadian gene expression in Drosophila. Cell 107: $567-578$.

McDonald MJ, Rosbash M, Emery P. 2001. Wild-type circadian rhythmicity is dependent on closely spaced $\mathrm{E}$ boxes in the Drosophila timeless promoter. Mol Cell Biol 21: 12071217.

Miyoshi K, Tsukumo H, Nagami T, Siomi H, Siomi MC. 2005. Slicer function of Drosophila Argonautes and its involvement in RISC formation. Genes \& Dev 19: 2837-2848.

Okamura K, Ishizuka A, Siomi H, Siomi MC. 2004. Distinct roles for Argonaute proteins in small RNA-directed RNA cleavage pathways. Genes \& Dev 18: 1655-1666.

Renn SC, Park JH, Rosbash M, Hall JC, Taghert PH. 1999. A pdf neuropeptide gene mutation and ablation of PDF neurons each cause severe abnormalities of behavioral circadian rhythms in Drosophila. Cell 99: 791-802.

Rothenfluh A, Abodeely M, Price JL, Young MW. 2000. Isolation and analysis of six timeless alleles that cause short or longperiod circadian rhythms in Drosophila. Genetics 156: 665675.

Rutila JE, Suri V, Le M, So WV, Rosbash M, Hall JC. 1998. CYCLE is a second bHLH-PAS clock protein essential for circadian rhythmicity and transcription of Drosophila period and timeless. Cell 93: 805-814.

Saini HK, Griffiths-Jones S, Enright AJ. 2007. Genomic analysis of human microRNA transcripts. Proc Natl Acad Sci 104: 17719-17724.

Sehgal A, Price J, Man B, Young M. 1994. Loss of circadian behavioral rhythms and per RNA oscillations in the Drosophila mutant timeless. Science 263: 1603-1606.

Sehgal A, Rothenfluh-Hilfiker A, Hunter-Ensor M, Chen Y, Myers M, Young MW. 1995. Circadian oscillations and autoregulation of timeless RNA. Science 270: 808-810.

Sire C, Moreno AB, Garcia-Chapa M, Lopez-Moya JJ, San Segundo B. 2009. Diurnal oscillation in the accumulation of Arabidopsis microRNAs, miR167, miR168, miR171 and miR398. FEBS Lett 583: 1039-1044.

Stoleru D, Peng Y, Agosto J, Rosbash M. 2004. Coupled oscillators control morning and evening locomotor behaviour of Drosophila. Nature 431: 862-868.

Stoleru D, Peng Y, Nawathean P, Rosbash M. 2005. A resetting signal between Drosophila pacemakers synchronizes morning and evening activity. Nature 438: 238-242.

Tomita J, Nakajima M, Kondo T, Iwasaki H. 2005. No transcription-translation feedback in circadian rhythm of KaiC phosphorylation. Science 307: 251-254.

Williams AJ, Su HS, Bernholm K, Field J, Sehgal A. 2001. A circadian output in Drosophila mediated by Neurofibromatosis-1 and Ras/MAPK. Science 293: 2251-2256.

Xu P, Vernooy SY, Guo M, Hay BA. 2003. The Drosophila microRNA Mir-14 suppresses cell death and is required for normal fat metabolism. Curr Biol 13: 790-795.

Xu S, Witmer PD, Lumayag S, Kovacs B, Valle D. 2007. MicroRNA (miRNA) transcriptome of mouse retina and identification 
miRNAs in the circadian clock of a sensory organ-specific miRNA cluster. I Biol Chem 282: 25053-25066.

Yang M, Lee JE, Padgett RW, Edery I. 2008. Circadian regulation of a limited set of conserved microRNAs in Drosophila. BMC Genomics 9: 83. doi: 10.1186/1471-2164-9-83.

Yu W, Zheng H, Houl JH, Dauwalder B, Hardin PE. 2006. PERdependent rhythms in CLK phosphorylation and E-box binding regulate circadian transcription. Genes \& Dev 20: 723-733. 


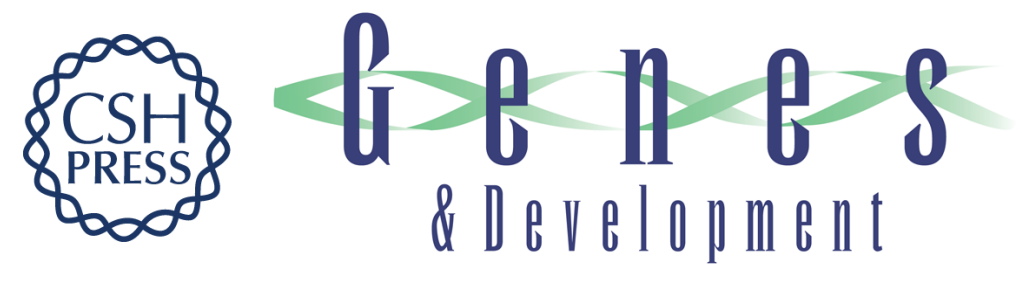

\section{A role for microRNAs in the Drosophila circadian clock}

Sebastian Kadener, Jerome S. Menet, Ken Sugino, et al.

Genes Dev. 2009, 23: originally published online August 20, 2009

Access the most recent version at doi:10.1101/gad.1819509

Supplemental

Material

References

License

Email Alerting Service
http://genesdev.cshlp.org/content/suppl/2009/08/21/gad.1819509.DC1

This article cites 55 articles, 22 of which can be accessed free at: http://genesdev.cshlp.org/content/23/18/2179.full.html\#ref-list-1

Receive free email alerts when new articles cite this article - sign up in the box at the top right corner of the article or click here.

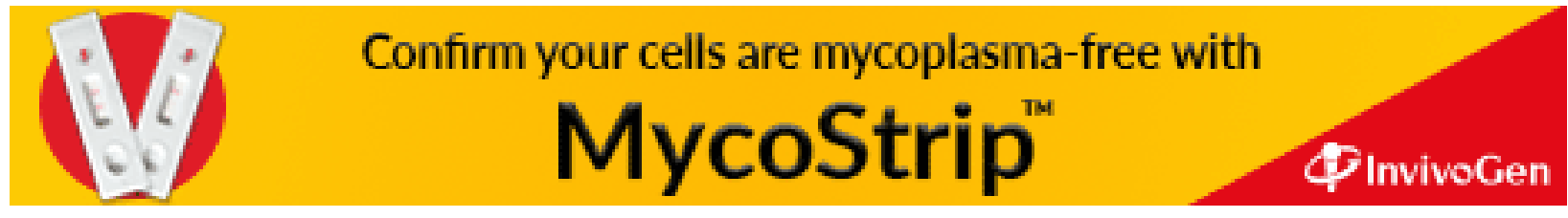

